\title{
Clinical course and outcomes of COVID-19 patients with a history of cerebrovascular disease: a retrospective study in Wuhan
}

\author{
Yang Bai ${ }^{1,2 \#}$, Yong Liang ${ }^{1 \#}$, Fang Wang ${ }^{3 \#}$, Ligang Chen ${ }^{1}$, Yulong Zhao ${ }^{1}$, Shoujie Zhao ${ }^{4}$, Yejing Zhu ${ }^{4}$, \\ Yushu Dong', Guobiao Liang ${ }^{1}$, Dongfeng Chen ${ }^{2}$, Lei Liu ${ }^{2,5}$ \\ ${ }^{1}$ Department of Neurosurgery, General Hospital of Northern Theater Command, Shenyang, China; ${ }^{2}$ Department of Gastroenterology, Daping \\ Hospital of Third Military Medical University, Chongqing, China; ${ }^{3}$ Department of Dermatology, The First Affiliated Hospital of Sun Yat-sen \\ University, Guangzhou, China; ${ }^{4}$ Department of General Surgery, Tangdu Hospital of Fourth Military Medical University, Xi'an, China; ${ }^{5}$ Department \\ of Gastroenterology, Tangdu Hospital of Fourth Military Medical University, Xi'an, China \\ Contributions: (I) Conception and design: G Liang, D Chen, L Liu; (II) Administrative support: None; (III) Provision of study materials or patients: \\ None; (IV) Collection and assembly of data: Y Bai, L Chen, Y Zhao, S Zhao, Y Zhao, Y Dong; (V) Data analysis and interpretation: Y Bai, Y Liang, \\ F Wang; (VI) Manuscript writing: All authors; (VII) Final approval of manuscript: All authors. \\ \#These authors contributed equally to this work. \\ Correspondence to: Guobiao Liang. Department of Neurosurgery, General Hospital of Northern Theater Command, Shenyang, China. Email: \\ liangguobiao6708@163.com; Lei Liu. Department of Gastroenterology, Daping Hospital of Third Military Medical University, Chongqing, China. \\ Email: liulei84207@163.com; Dongfeng Chen. Department of Gastroenterology, Daping Hospital of Third Military Medical University, Chongqing, \\ China. Email: chendf@tmmu.edu.cn.
}

Background: Data on patients with coronavirus disease 2019 (COVID-19) who have pre-existing cerebrovascular disease (CVD) are scarce. This study set out to describe the clinical course and outcomes of these patients.

Methods: This single-center retrospective study was performed at Huoshenshan Hospital in Wuhan, China. Patients with confirmed COVID-19 who had pre-existing CVD (N=69) were identified. COVID-19 patients without CVD were randomly selected and matched by age and sex to the patients with CVD. Clinical data were analyzed and compared between the 2 groups. The composite endpoint included intensive care unit admission, use of mechanical ventilation, and death. Multivariable Cox regression analyses with control for medical comorbidities were used to examine the relationship between pre-existing CVD and clinical outcome of COVID-19.

Results: Compared with patients without CVD, patients with pre-existing CVD were more likely to present with unapparent symptoms at first; however, at admission, these patients tended to be in a severer condition than those without CVD, with more underlying hypertension and diabetes. The levels of interleukin-6, creative kinase $\mathrm{MB}$, aspartate transaminase, and creatinine, as well as prothrombin time, were also markedly higher in patients with CVD. Patients with pre-existing CVD were more likely to develop multi-organ dysfunction, deteriorate to critical condition, and yield poorer clinical outcomes than patients without CVD. Concerning therapeutics, greater proportions of patients with pre-existing CVD required mechanical ventilation, higher-order anti-bacterials, and drugs targeting underlying diseases and complications. In the multivariable analysis, pre-existing CVD was significantly associated with a poor clinical outcome.

Conclusions: Patients with a history of CVD are more vulnerable to an over-activated inflammatory response and subsequent multi-organ dysfunction, resulting in a poor clinical outcome. Close monitoring is advisable for these patients.

Keywords: Coronavirus disease 2019 (COVID-19); severe acute respiratory syndrome coronavirus-2 (SARS$\mathrm{CoV}-2$ ); cerebrovascular disease (CVD); clinical course; clinical outcome 
Submitted Apr 20, 2021. Accepted for publication May 28, 2021.

doi: $10.21037 /$ atm-21-2237

View this article at: http://dx.doi.org/10.21037/atm-21-2237

\section{Introduction}

The coronavirus disease 2019 (COVID-19) pandemic, caused by severe acute respiratory syndrome coronavirus- 2 (SARS-CoV-2), is continuing to rage on around the globe (1). The World Health Organization (WHO) reported that, as of April 2021, there had been over 3.1 million deaths from the disease, with older people with underlying diseases, such as hypertension, diabetes, and cardiovascular disease, impacted hardest $(2,3)$. Cerebrovascular disease (CVD) is another common senile disease, and cerebrovascular accidents as neurological complications of COVID-19 have been put much emphasis in prior studies. Regarding CVD as a comorbidity of COVID-19 (4), several retrospective studies (5-11) and meta-analyses (12-15) have confirmed that pre-existing CVD is associated with COVID-19 severity and mortality. However, little is known of the clinical characteristics of COVID-19 patients with preexisting CVD, or of the impact of pre-existing CVD on the clinical course and outcomes of the disease.

Previous reports have shown that COVID-19 patients with pre-existing CVD are older, are more likely to be male, and have more comorbidities than those without CVD (5-8). However, the impact of discrepancies in these demographical features between patients with and without pre-existing CVD was not considered in these studies. To explore the impact of CVD pathophysiology on the clinical course of COVID-19, clinical data of COVID-19 patients with pre-existing CVD treated at Huoshenshan Hospital (Wuhan, China) and age- and sex-matched non-CVD COVID-19 patients selected from the same cohort were compared. The association of pre-existing CVD with the clinical outcome of COVID-19 was also examined via a multivariable Cox regression analysis. We present the following article in accordance with the STROBE reporting checklist (available at http://dx.doi. org/10.21037/atm-21-2237).

\section{Methods}

\section{Study participants}

Consecutive patients admitted to Huoshenshan Hospital between 5 February and 15 March, 2020 were included in this retrospective cohort study. This hospital, located in Wuhan in the province of Hubei, was assigned the responsibility of treating patients with COVID-19 by the Chinese government. The inclusion and exclusion criteria for patients are shown in Figure 1.

All confirmed COVID-19 patients with pre-existing CVD included in this study had a clear diagnosis of CVD by a physician on the report of the patients themselves or their family members, as well as their electronic medical records. Compared with the overall study population, patients with pre-existing CVD were significantly older [median (IQR), 71.0 (67.0-81.0) vs. 60.0 (49.0-68.0); $\mathrm{P}<0.001]$, and a higher proportion were male $(56.5 \%$ vs. $51.1 \% ; \mathrm{P}<0.001)$. Older age and male sex have been demonstrated to be associated with in-hospital death among patients with COVID-19 $(16,17)$. Thus, to adjust for age and sex, an age- $( \pm 2$ years $)$ and sex-matched COVID-19 patient without CVD was randomly selected for each patient in the CVD group, using a method similar to ones reported previously $(18,19)$. Whenever more than one non-CVD patient was available for a patient with CVD, a match was randomly selected from the patients available. This study was conducted in accordance with the Declaration of Helsinki (as revised in 2013) and approved by the National Health Commission of China and the Institutional Review Board in Huoshenshan Hospital (approval No. K202101-02). The requirement to obtain written informed consent from patients with emerging infectious diseases was waived by the ethics committee of the designated hospital.

\section{Data collection}

All data were extracted from patients' electronic medical records by 2 investigators and then independently reviewed by 2 analysts. According to a previous observational study (20), clinical data were collected in detail, which included: demographic information (age, sex, and comorbidities); onset symptoms (e.g., fever, cough, dyspnea, and myalgia); vital signs (blood pressure, heart rate, respiratory rate, and body temperature); laboratory data (i.e., infection-related indices, blood routine test, coagulation function test, myocardial injury markers, liver function indices, kidney function indices, electrolytes, and glucose); and complications (e.g., respiratory 


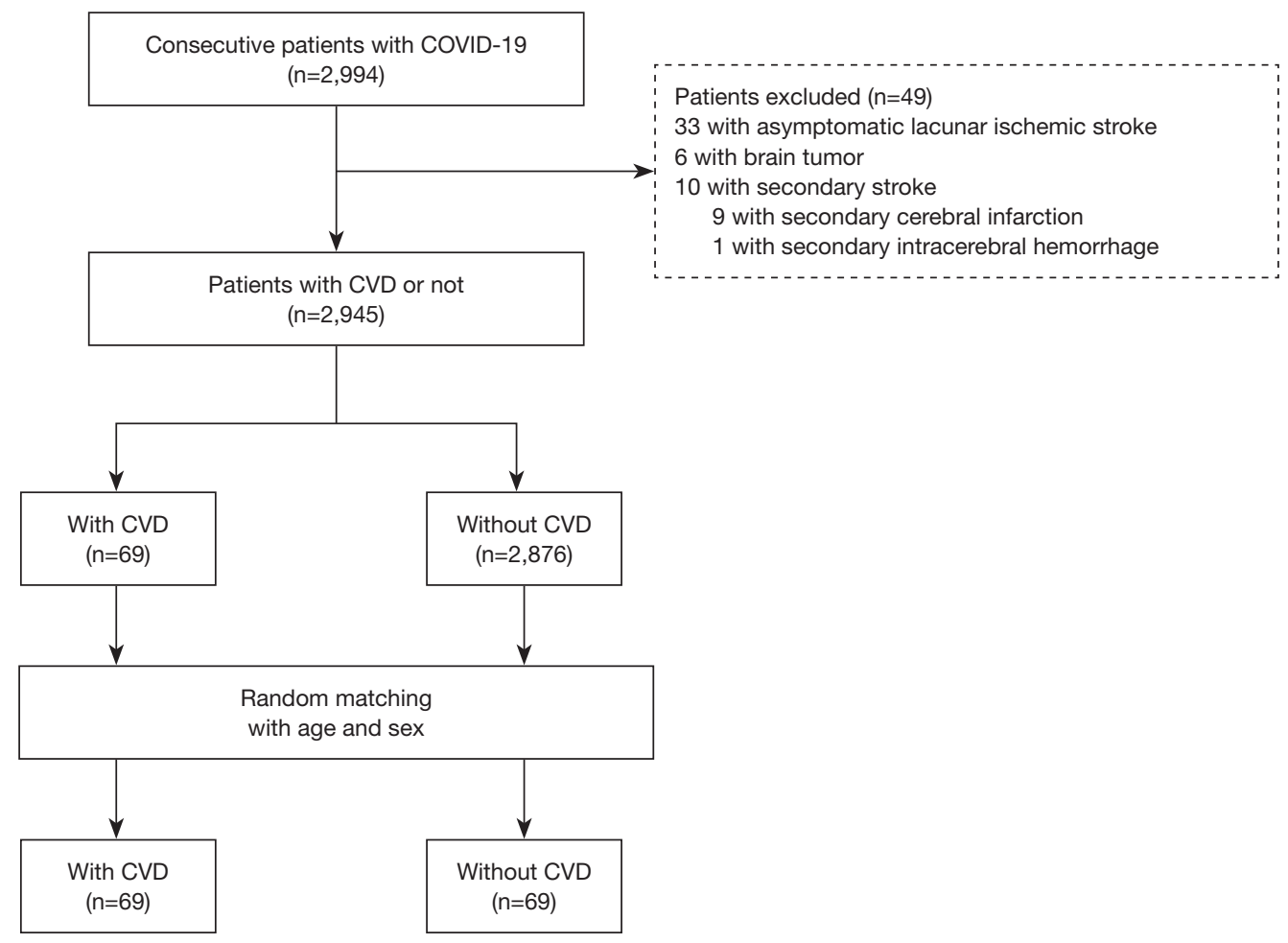

Figure 1 Flowchart of patient recruitment.

failure and septic shock).

Additionally, clinical information concerning therapeutics were also collected. According to the WHO's interim guidelines (21), all patients received individualized systematic treatment, including antivirals, oxygen support, secondary infection control, immunomodulators, and multiorgan support. As the key therapeutic approach, oxygen therapy was given through normal or high-flow nasal cannulas, non-invasive or invasive mechanical ventilation, or extracorporeal membrane oxygenation (ECMO). For patients with a history of CVD, individualized therapy including antiplatelets, anti-coagulation agents, antihypertensives, antidiabetic agents, or brain-protective agents were administered according to the relevant guidelines $(22,23)$.

In this study, a composite endpoint consisting of unfavorable clinical outcomes including admission to the intensive care unit (ICU), use of mechanical ventilation, and death was adopted as the primary outcome. Secondary outcomes included the occurrence of disease progression, disease deterioration, viral clearance, and death. All patients were followed up to discharge from hospital after recovery, or death. The durations of onset of COVID-19 symptoms to hospital admission, disease progression, disease deterioration, death, the occurrence of the composite endpoint, and viral clearance, were recorded, respectively.

\section{Definitions}

Cases of COVID-19 were diagnosed on the basis of the WHO's interim guidance (21). Disease severity was defined in accordance with the guidelines for the diagnosis and management of COVID-19 ( $6^{\text {th }}$ edition) released by the National Health Commission of China (24). Disease progression and disease deterioration were defined as the exacerbation of disease from non-severe types (including mild and moderate types) to severe and critical types, respectively. Viral clearance was considered when negative nucleic acid results of respiratory tract specimens were produced 3 times consecutively with sampling intervals of more than 24 hours.

The occurrence of complications was confirmed according to the following criteria. Anemia was diagnosed based on hemoglobin $<110 \mathrm{~g} / \mathrm{L}$. Myocardial injury was reported if the serum levels of cardiac troponin I (cTnI) or creatine kinase isoenzyme (CKMB) exceeded the upper 
limit of normal (ULN) (25). The diagnosis of liver injury was made based on an alanine transaminase (ALT) or aspartate aminotransferase (AST) level more than 3-fold the ULN, or a total bilirubin (TBIL) level more than 2-fold the ULN (26). Hypoproteinemia was defined by a serum albumin level less than $25 \mathrm{~g} / \mathrm{L}$ (16). Acute kidney injury (AKI) was defined by an increase in serum creatinine $(\mathrm{Cr})$ levels to more than 1.5 times the baseline (27). Sepsisinduced coagulopathy (SIC) and disseminated intravascular coagulation (DIC) were diagnosed according to the International Society of Thrombosis and Haemostasis (ISTH) scoring systems $(28,29)$. Cardiac insufficiency was defined as a serum level of brain natriuretic peptide (BNP) exceeding the normal range combined with the presence of associated symptoms, such as dyspnea, orthopnea, and edema of the lower extremity (30). Acute respiratory distress syndrome (ARDS) was diagnosed according to the Berlin definition (31). Respiratory failure was defined by an arterial partial pressure of oxygen of less than $60 \mathrm{mmHg}$. Multiple organ dysfunction syndrome (MODS) was diagnosed on the basis of the multiple organ dysfunction score (32). Shock was defined in accordance with the 2016 Third International Consensus Definition for Sepsis and Septic Shock (33).

\section{Statistical analysis}

No imputation was made for variables with missing data. Quantitative data with non-normal distribution were expressed as medians [interquartile ranges (IQRs)] and statistically compared using the Mann-Whitney U nonparametric test. Percentages (\%) of enumeration data were calculated and compared using the $\chi^{2}$ test or Fisher's exact test. Survival curves were plotted employing the KaplanMeier method with the log-rank test.

For the assessment of whether pre-existing CVD was an independent risk factor for the primary outcome, Cox regression analyses were carried out. Considering the death toll was not large in our study, and to avoid overfitting in the multivariable model, 3 variables (age, CVD, and malignancy) were chosen for the multivariable model. All variables included were based on their clinical and scientific merits, previous findings, and the results of univariable analyses. Variables (i.e., atrial fibrillation and chronic kidney disease) were excluded from the Cox regression models if the number of events was deemed too small. All statistical analyses were performed with SPSS software (version 22.0, IBM Corp). $\mathrm{P}<0.05$ was considered to indicate statistical significance.

\section{Results}

\section{Identification of patients with pre-existing CVD}

Figure 1 depicts the flowchart of participant selection. A total of 2,994 consecutive hospitalized patients with confirmed COVID-19 in the medical record system were screened from 5 February to 15 March, 2020. Among these cases, 33 patients with asymptomatic lacunar ischemic stroke (diagnosed in previous health examinations), 10 patients with secondary stroke (diagnosed after the occurrence of COVID-19), and 6 patients with brain tumor were excluded. Among the 2945 cases remaining, 69 patients with a history of CVD were identified, including 56 cases with cerebral infarction, 8 cases with symptomatic lacunar ischemic stroke, 4 cases with intracerebral hemorrhage, and 1 case with subarachnoid hemorrhage (Figure 2A). Of these 69 patients, 9 exhibited no residual symptoms on admission, whereas the others presented with neurological sequelae such as limb dyskinesia, cognitive disorder, aphasia, loss of self-sufficiency, sensory disorder, bulbar paralysis, and prosopoplegia (Figure $2 \mathrm{~B}$ ). The median interval from the diagnosis of CVD to admission was 6 years (IQR, $1-10$ years), and there were 13 patients who had been newly diagnosed with CVD within the previous 3 months.

\section{Demographic and clinical features}

Baseline demographic and clinical features of the COVID-19 patients with pre-existing CVD and matched patients without CVD are shown in Table 1. There was no difference in the duration from the onset of COVID-19 symptoms to admission between the 2 groups [22.0 (7.0 $40.5)$ vs. 21.0 (14.0-36.0) days; $\mathrm{P}=0.427]$. Patients with pre-existing CVD had a higher prevalence of comorbid hypertension $(75.4 \%$ vs. $43.5 \%)$ and diabetes $(34.8 \%$ vs. $11.6 \%$ ) than patients without CVD (all P values $<0.01$ ). Interestingly, in the early stage of the disease, patients with CVD were more likely to be asymptomatic ( $17.4 \%$ vs. $2.9 \%$; $\mathrm{P}=0.005$ ) and exhibited fewer systematic symptoms (i.e., chill, myalgia and fatigue; all $\mathrm{P}$ values $<0.05$ ) and respiratory symptoms (i.e., dry cough and dyspnea; all $\mathrm{P}$ values $<0.01$ ). Despite having less obvious symptoms, patients with CVD were more likely than those without CVD to be in a severe condition $(43.5 \%$ vs. $26.1 \% ; \mathrm{P}=0.032)$ on admission. No significant difference was found in vital signs on admission 
A

Disease types of CVD

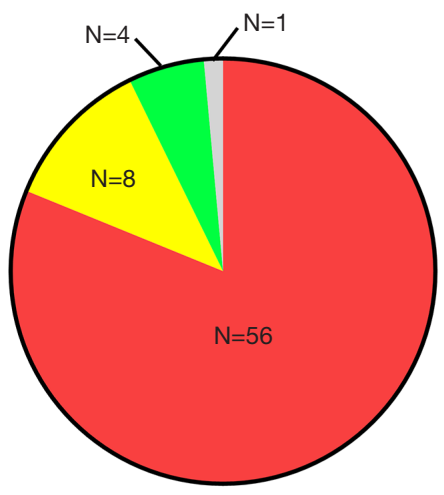

$\square$ Cerebral infarction

$\square$ Symptomatic lacunar ischemic stroke

$\square$ Intracerebral hemorrhage

$\square$ Subarachnoid hemorrhage

B

Current sequelae of CVD

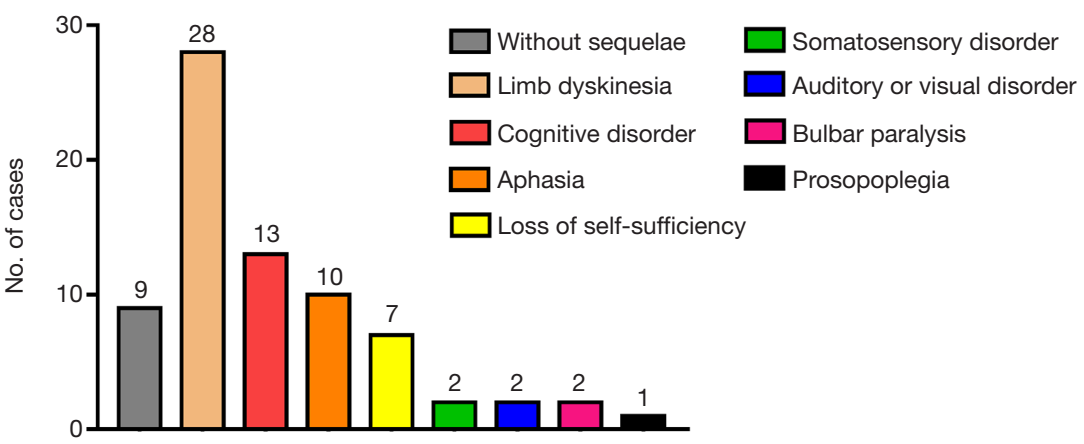

Figure 2 Disease type (A) and current neurological manifestations (B) of 69 patients with cerebrovascular diseases.

Table 1 Demographic and clinical features of matched COVID-19 patients with and without CVD

\begin{tabular}{lccc}
\hline Variable & Patients with CVD $(\mathrm{n}=69)$ & Patients without CVD $(\mathrm{n}=69)$ & $\mathrm{P}^{\mathrm{a}}$ \\
\hline Age, y & $71.0(67.0-81.0)$ & $71.0(66.5-81.0)$ & 0.811 \\
Male, No. (\%) & $39(56.5)$ & $39(56.5)$ & 1.000 \\
Symptom onset to admission, d & $22.0(7.0-40.5)$ & $21.0(14.0-36.0)$ & 0.427 \\
Comorbidity, No. (\%) & & $30(43.5)$ & $0.000^{*}$ \\
Hypertension & $52(75.4)$ & $8(11.6)$ & $0.001^{*}$ \\
Diabetes & $24(34.8)$ & $10(14.5)$ & 0.500 \\
CHD & $14(20.3)$ & $1(1.4)$ & $0.362^{\mathrm{a}}$ \\
AF & $4(5.8)$ & $7(10.1)$ & 1.000 \\
COPD & $8(11.6)$ & $1(1.4)$ & $1.000^{\mathrm{a}}$ \\
CKD & $2(2.9)$ & $4(5.8)$ & $1.000^{\mathrm{a}}$ \\
Malignancy & $5(7.2)$ & $51(73.9)$ & $0.032^{*}$ \\
Disease classification, No. (\%) & $39(56.5)$ & & \\
Non-severe group & & & \\
\hline
\end{tabular}

Table 1 (continued) 
Table 1 (continued)

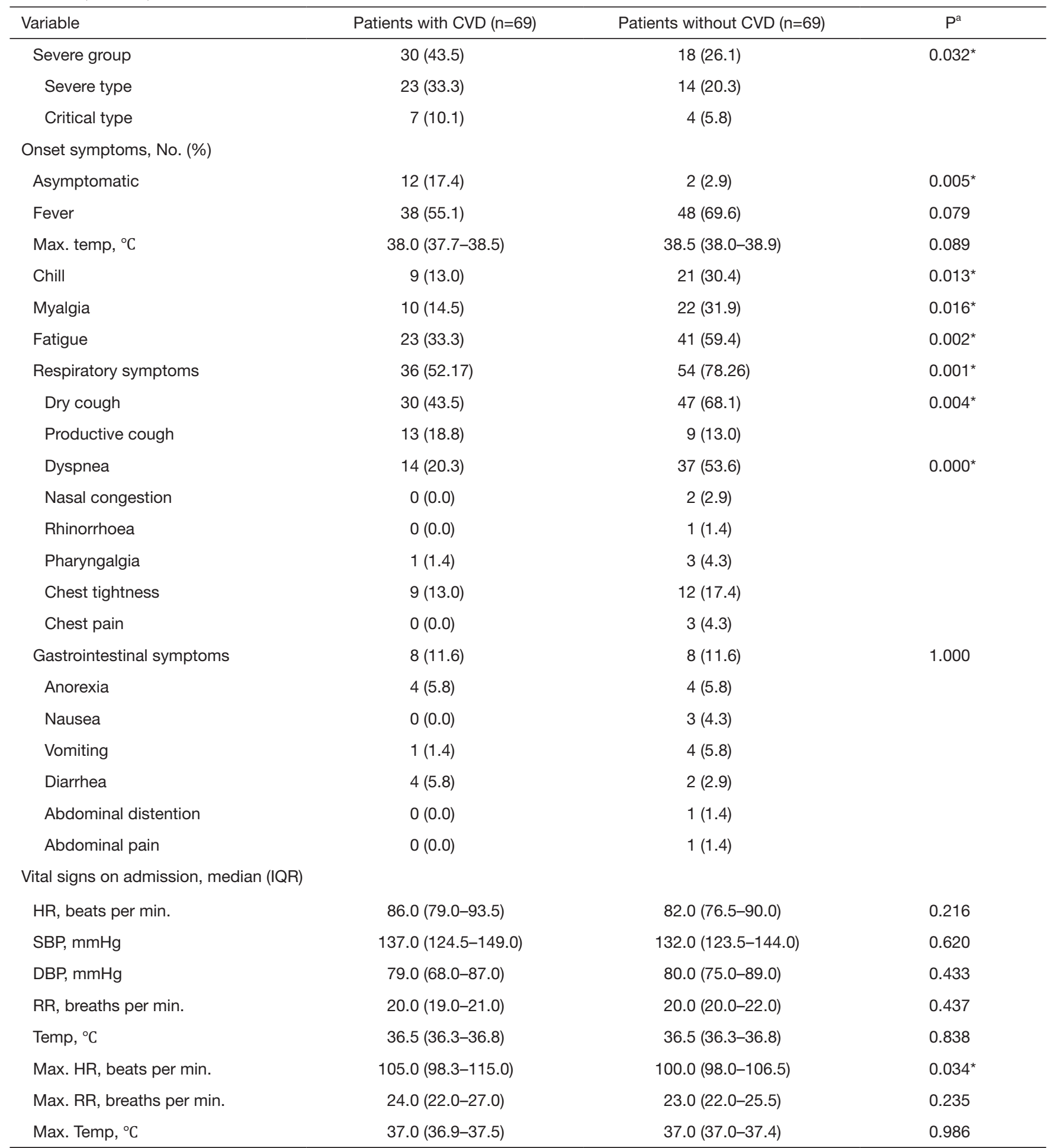

${ }^{a}$, compared by Fisher's exact test. * $\mathrm{P}<0.05$. AF, atrial fibrillation; CHD, coronary heart disease; CKD, chronic kidney disease; COPD, chronic obstructive pulmonary disease; CVD, cerebrovascular disease; DBP, diastolic blood pressure; HR, heart rate; Max., maximum; RR, respiratory rate; SBP, systolic blood pressure; temp., temperature. 
between patients with and without CVD; however, during hospitalization, those with CVD exhibited higher peak heart rates $[105.0(98.3-115.0)$ vs. $100.0(98.0-106.5)$ beats per min; $\mathrm{P}<0.05)]$.

\section{Laboratory findings}

Initial laboratory parameters of the patients are shown in Table 2. In terms of infection-related indices, patients with pre-existing CVD presented with significantly higher interleukin-6 (IL-6) [8.7 (3.6-36.7) vs. 2.8 (1.5-5.4) pg/mL] than those without CVD. Regarding coagulation function, patients with CVD exhibited prolonged prothrombin time (PT) and thrombin time (TT), as well as an increased international normalized ratio (INR) (all $\mathrm{P}<0.05)$. Also, patients with CVD displayed higher levels of serum CKMB, myoglobin, AST, blood urea nitrogen, $\mathrm{Cr}$, cystatin-c, urine red blood cells, and urine protein (all $\mathrm{P}<0.05$ ).

Apart from initial laboratory indices, during hospitalization, the peak or lowest values of some parameters which are of importance to the diagnosis of complications were also recorded and analyzed. Patients with CVD had higher peak levels of IL-6 [8.8 (3.8-77.8) vs. $3.1(1.5-8.2) \mathrm{pg} / \mathrm{mL}]$, procalcitonin (PCT) $[0.08$ (0.04-0.49) vs. $0.05(0.04-0.08) \mathrm{ng} / \mathrm{mL}]$, CKMB [13.9 (9.2-20.3) vs. $9.5(7.4-13.2) \mathrm{mg} / \mathrm{L}], \mathrm{c} \operatorname{TnI}[0.01(0.01-$ $0.06)$ vs. $0.01(0.01-0.01) \mathrm{ng} / \mathrm{mL}]$, and AST [25.8 (18.543.1) vs. 20.4 (16.9-32.8) IU/L], and a longer PT [13.7 $(12.7-15.1)$ vs. $12.8(12.3-13.7) \mathrm{s}$, than patients without CVD (all $\mathrm{P}<0.05)$.

\section{Complications and treatments}

As shown in Table 3, in comparison to patients without CVD, those with pre-existing CVD had a higher likelihood of developing ARDS (14.5\% vs. $4.3 \%$; $\mathrm{P}<0.05)$. Furthermore, 23 (33.3\%), 19 (27.5\%), 18 (26.1\%), and 16 (23.2\%) patients with CVD had AKI, hypoproteinemia, SIC, and myocardial injury, respectively, which were significantly higher than those seen among patients without CVD $(17.4 \%, 13.0 \%, 11.6 \%$, and $5.8 \%)$, respectively (all $\mathrm{P}<0.05$ ).

Regarding treatments (Table 4), patients with pre-existing CVD required more routine therapies for comorbidities than patients without CVD, including antihypertensives (71.0\% vs. $46.4 \%)$, antidiabetic agents (34.8\% vs.11.6\%), antilipemics $(37.7 \%$ vs. $14.5 \%)$, amiodarone $(8.7 \%$ vs. $0.0 \%$ ), and antiplatelet agents $(42.0 \%$ vs. $23.2 \%$ ) (all
$\mathrm{P}<0.05)$. Importantly, patients with CVD were also more likely to need sedatives and analgesics (13.0\% vs. $2.9 \%)$, mechanical ventilation (17.4\% vs. 5.8\%), and high-order antibacterials $(30.4 \%$ vs. $8.7 \%)$ (all $\mathrm{P}<0.05)$, but not immunomodulators (all $\mathrm{P}>0.05$ ). The use of therapies to prevent or treat complications, including anticoagulants (23.2\% vs. $8.7 \%)$, hepatic protectants (21.7\% vs. $8.7 \%)$, and albumin transfusion $(31.9 \%$ vs. $14.5 \%)$ was also significantly higher in patients with than without CVD (all $\mathrm{P}<0.05$ ). However, the use of anti-asthmatic agents was lower among patients with CVD (11.6\% vs. $29.0 \% ; \mathrm{P}<0.05)$.

\section{Outcomes}

As shown in Table 3, patients with pre-existing CVD were more likely than those without CVD to experience disease deterioration $(20.3 \%$ vs. $7.2 \%$; $\mathrm{P}<0.05)$ but not disease progression $(\mathrm{P}>0.05)$. Despite no significant difference existing in the length of hospital stay between the 2 groups $(\mathrm{P}>0.05)$, patients with CVD had worse outcomes, with higher rates of admission to the ICU (17.4\% vs. $5.8 \%)$, use of mechanical ventilation (17.4\% vs. $5.8 \%$ ), and death (11.6\% vs. $2.9 \%)($ all $\mathrm{P}<0.05)$. The Kaplan-Meier survival curves also showed that patients with CVD had significantly escalated risks of disease deterioration [hazard ratio (HR), 3.104; 95\% confidence interval (CI), 1.118-8.619; Log-rank $\mathrm{P}=0.021$ ], death (HR, 4.378; 95\% CI, 0.929-20.621; Logrank $\mathrm{P}=0.041$ ), and unfavorable outcomes (HR, 3.017; $95 \%$ CI, 1.075-8.466; Log-rank $\mathrm{P}=0.027$ ) compared to their non-CVD counterparts (Figure 3). However, no significant difference in disease progression or viral clearance was observed between the 2 groups (Figure 3).

In the initial univariate Cox regression analyses of demographic variables, CVD was identified as a changed risk factor (HR, 3.017; 95\% CI, 1.075-8.466; $\mathrm{P}=0.036$ ) for an unfavorable outcome among the patients in the cohort. Thereafter, a multivariate model including age, CVD, and malignancy was established employing a forward stepwise approach, which indicated that CVD was an independent risk factor (HR, 3.155; 95\% CI, 1.121-8.878; $\mathrm{P}=0.030)$ for an unfavorable clinical outcome of COVID-19 (Table 5).

\section{Discussion}

The present study analyzed the characteristics of COVID-19 patients with pre-existing CVD and sex- and age-matched COVID-19 patients without CVD. Among these patients with COVID-19, CVD was identified as an 
Table 2 Laboratory results of matched COVID-19 patients with and without CVD

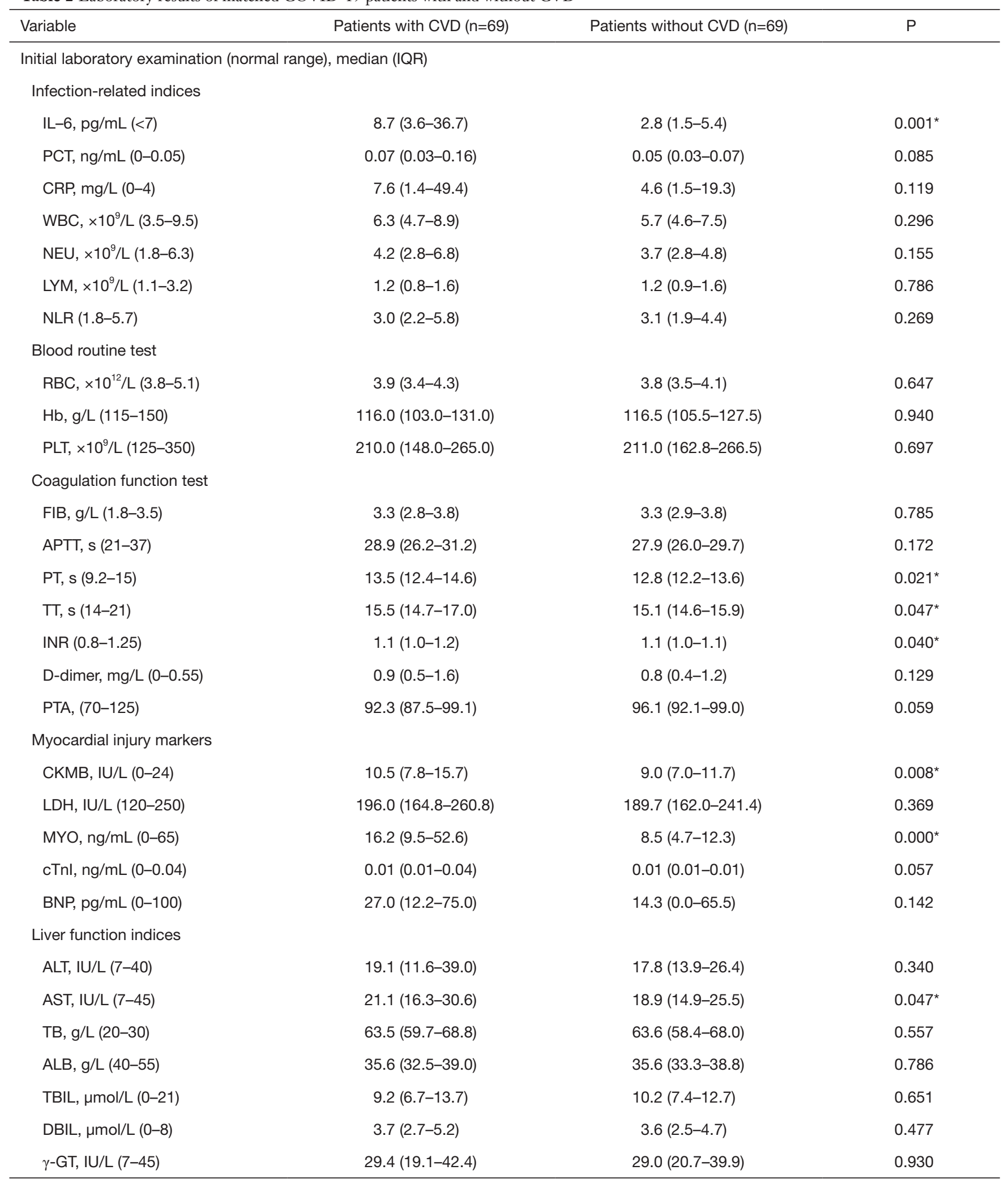

Table 2 (continued) 
Table 2 (continued)

\begin{tabular}{|c|c|c|c|}
\hline Variable & Patients with CVD $(n=69)$ & Patients without CVD $(n=69)$ & $\mathrm{P}$ \\
\hline BUN, mmol/L (3.1-8.8) & $5.7(4.4-8.9)$ & $4.5(3.6-6.1)$ & $0.001^{*}$ \\
\hline $\mathrm{Cr}, \mu \mathrm{mol} / \mathrm{L}(41-81)$ & $77.3(59.3-94.7)$ & $66.6(56.3-78.2)$ & $0.032^{*}$ \\
\hline CysC, mg/L (22-29) & $1.2(1.0-1.5)$ & $1.0(0.9-1.2)$ & $0.000^{*}$ \\
\hline UPRO (0) & $0.0(0.0-0.5)$ & $0.0(0.0-0.0)$ & $0.001^{*}$ \\
\hline \multicolumn{4}{|l|}{ Electrolytes and glucose } \\
\hline $\mathrm{Na}^{+}, \mathrm{mmol} / \mathrm{L}(137-147)$ & $141.2(138.1-143.8)$ & $141.7(139.0-143.7)$ & 0.978 \\
\hline $\mathrm{K}^{+}, \mathrm{mmol} / \mathrm{L}(3.5-5.3)$ & $4.3(3.8-4.7)$ & $4.3(4.0-4.5)$ & 0.624 \\
\hline Glu, mmol/L (3.9-6.1) & $5.5(5.0-6.5)$ & $4.9(4.7-5.8)$ & $0.002^{*}$ \\
\hline \multicolumn{4}{|c|}{ Peak/lowest value during hospitalization (normal range), median (IQR) } \\
\hline Max. CRP, mg/L (0-4) & $12.3(1.2-71.6)$ & $5.8(1.9-30.7)$ & 0.088 \\
\hline Max. PCT, ng/mL (0-0.05) & $0.08(0.04-0.49)$ & $0.05(0.04-0.08)$ & $0.030^{*}$ \\
\hline Max. IL-6, pg/dL $(<0.07)$ & $8.8(3.8-77.8)$ & $3.1(1.5-8.2)$ & $0.001^{*}$ \\
\hline Max. WBC, $\times 10^{9} / L(3.5-9.5)$ & $7.1(5.5-11.3)$ & $6.5(5.1-8.1)$ & 0.074 \\
\hline Min. Hb, g/L (115-150) & $111.0(87.0-121.5)$ & $112.0(99.5-123.0)$ & 0.158 \\
\hline Min. PLT, ×109/L (125-350) & $190.0(132.5-232.5)$ & $199.0(160.5-236.5)$ & 0.399 \\
\hline Max. cTnl, ng/mL (0-0.04) & $0.01(0.01-0.06)$ & $0.01(0.01-0.01)$ & $0.046^{*}$ \\
\hline Max. BNP, pg/mL (0-100) & $44.3(12.9-133.1)$ & $18.2(0.0-70.0)$ & 0.091 \\
\hline Max. AST, IU/L (7-45) & $25.8(18.5-43.1)$ & $20.4(16.9-32.8)$ & $0.034^{*}$ \\
\hline Min. ALB, g/L (40-55) & $34.0(29.3-37.4)$ & $35.3(32.6-37.8)$ & 0.193 \\
\hline Max. TBIL, $\mu \mathrm{mol} / \mathrm{L}(0-21)$ & $10.9(8.0-16.0)$ & $11.4(8.5-14.0)$ & 0.981 \\
\hline Max. Cr, $\mu \mathrm{mol} / \mathrm{L}$ (41-81) & $79.1(63.2-110.9)$ & $68.2(57.7-79.0)$ & $0.016^{*}$ \\
\hline
\end{tabular}

*, P<0.05. ALB, albumin; ALT, alanine transaminase; APTT, activated partial thromboplastin time; AST, aspartate transaminase; BUN, blood urea nitrogen; BNP, brain natriuretic peptide; CKMB, creative kinase MB; Cr, creatinine; CRP, C-reactive protein; cTnl, cardiac tropinin I; CVD, cerebrovascular disease; CysC, cystatin C; DBIL, direct bilirubin; FIB, fibrinogen; Glu, glucose; Hb, hemoglobin; IL-6, interleukin-6; INR, international normalized ratio; LDH, lactate dehydrogenase; LYM, lymphocyte; MYO, myoglobin; NEU, neutrophil; NLR, neutrophil-tolymphocyte ratio; PCT, procalcitonin; PLT, blood platelet; PT, prothrombin time; PTA, prothrombin activity; RBC, red blood cell; TBIL, total bilirubin; TT, thrombin time; UPRO, urine protein; URBC, urine red blood cell; WBC, white blood cell. 
Table 3 Complications and outcomes of matched COVID-19 patients with and without CVD

\begin{tabular}{|c|c|c|c|}
\hline Variable & Patients with CVD $(n=69)$ & Patients without CVD $(n=69)$ & $\mathrm{P}^{\mathrm{a}}$ \\
\hline GIB & $5(7.2)$ & $1(1.4)$ & $0.210^{\mathrm{a}}$ \\
\hline Anemia & $33(47.8)$ & $31(44.9)$ & 0.733 \\
\hline Myocardial injury & $16(23.2)$ & $4(5.8)$ & $0.004^{*}$ \\
\hline Hypoproteinemia & $19(27.5)$ & $9(13.0)$ & $0.038^{*}$ \\
\hline $\mathrm{AKI}$ & $23(33.3)$ & $12(17.4)$ & $0.031^{*}$ \\
\hline $\mathrm{SIC}$ & $18(26.1)$ & $8(11.6)$ & $0.029^{*}$ \\
\hline Cardiac insufficiency & $4(5.8)$ & $0(0.0)$ & $0.128^{\mathrm{a}}$ \\
\hline Respiratory failure & $10(14.5)$ & $4(5.8)$ & 0.091 \\
\hline MODS & $3(4.3)$ & $1(1.4)$ & $0.612^{\mathrm{a}}$ \\
\hline Septic shock & $6(8.7)$ & $1(1.4)$ & $0.121^{\mathrm{a}}$ \\
\hline \multicolumn{4}{|l|}{ Outcomes, No. (\%) } \\
\hline Length of stay, median (IQR), d & $12.0(7.0-18.0)$ & $13.0(7.0-19.5)$ & 0.435 \\
\hline Disease progression & $38(55.1)$ & $35(50.7)$ & 0.609 \\
\hline Disease deterioration & $14(20.3)$ & $5(7.2)$ & $0.026^{*}$ \\
\hline Composite endpoint & $13(18.8)$ & $5(7.2)$ & $0.043^{*}$ \\
\hline Disease deterioration & $36.0(24.5-47.5)$ & $39.0(29.5-49.0)$ & 0.185 \\
\hline Discharge & $37.0(25.5-49.5)$ & $39.0(31.0-50.5)$ & 0.354 \\
\hline Composite endpoint & $35.0(23.5-47.5)$ & $39.0(29.5-49.0)$ & 0.095 \\
\hline
\end{tabular}

a, compared by Fisher's exact test. *, P<0.05. AKI, acute kidney injury; ARDS, acute respiratory distress syndrome; CVD, cerebrovascular disease; DIC, disseminated intravascular coagulation; GIB, gastrointestinal bleeding; ICU, intensive care unit; MODS, multiple organ dysfunction syndrome; SIC, sepsis-induced coagulopathy.

independent predictor of adverse outcomes. Among patients hospitalized with COVID-19 in Huoshenshan Hospital between 5 February and 15 March, 2020, the prevalence of CVD was $2.3 \%(69 / 2,994)$. Compared with COVID-19 patients without CVD, those with pre-existing CVD were more likely to have underlying diseases, and, although they tended to present with unapparent initial symptoms, they had severer COVID-19 on admission. Furthermore, during the course of their disease, patients with pre-existing CVD were more likely to have severer secondary infection, develop ARDS and subsequent multi-organ dysfunction, deteriorate to a critical condition, and ultimately yield a worse outcome. Correspondingly, higher proportions of patients with pre-existing CVD required higher-order anti- 
Table 4 Treatment of matched COVID-19 patients with and without CVD

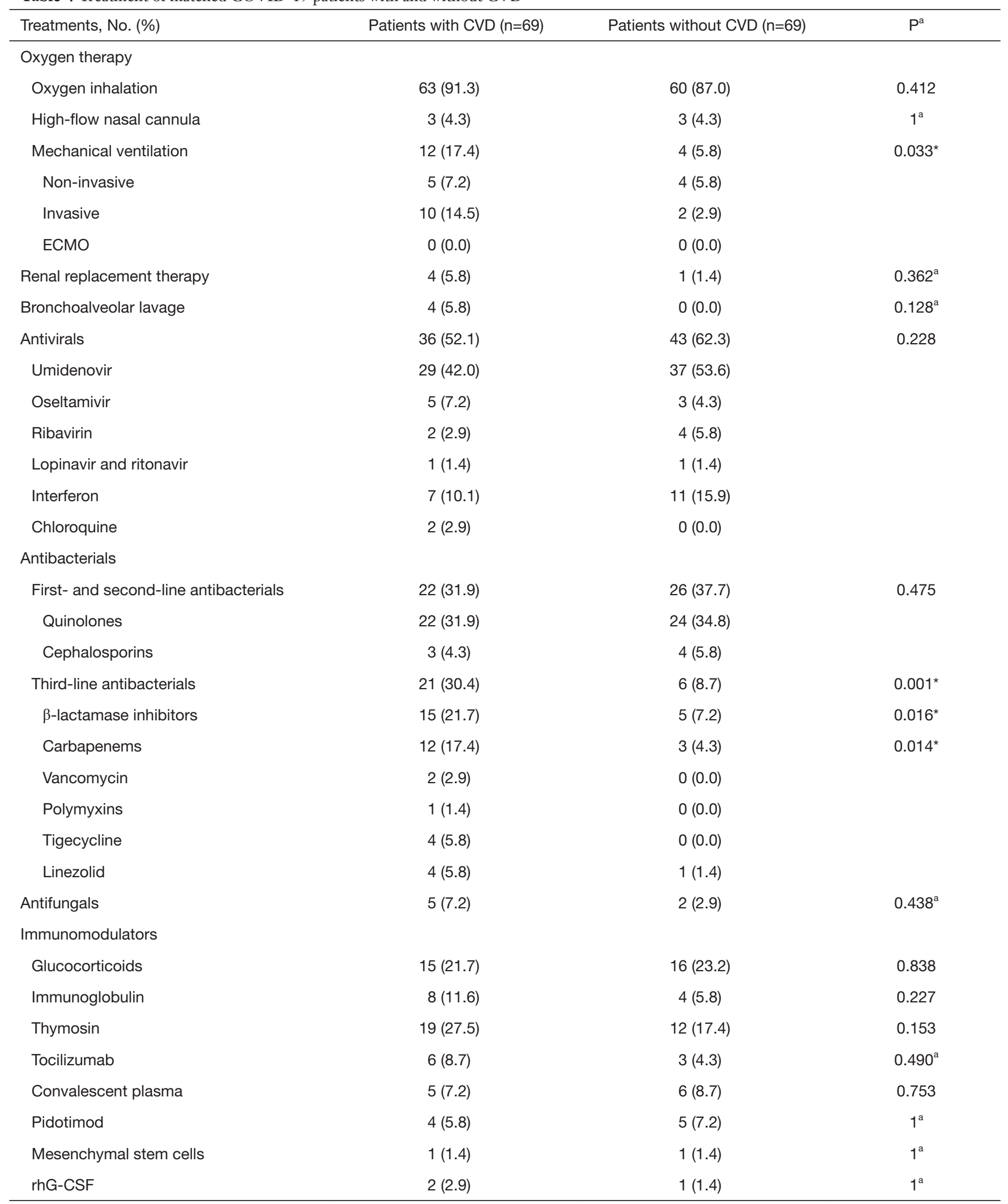

Table 4 (continued) 
Table 4 (continued)

\begin{tabular}{|c|c|c|c|}
\hline Treatments, No. (\%) & Patients with CVD (n=69) & Patients without CVD ( $\mathrm{n}=69)$ & $P^{a}$ \\
\hline Diuretics & $5(7.2)$ & $0(0.0)$ & \\
\hline RAAS inhibitors & $12(17.4)$ & $5(7.2)$ & \\
\hline Beta-blockers & $13(18.8)$ & $9(13.0)$ & \\
\hline Urapidil & $2(2.9)$ & $0(0.0)$ & \\
\hline Nitroglycerin & $1(1.4)$ & $0(0.0)$ & \\
\hline Antidiabetics & $24(34.8)$ & $8(11.6)$ & $0.001^{*}$ \\
\hline Insulin & $12(17.4)$ & $5(7.2)$ & \\
\hline \multicolumn{4}{|c|}{ Drugs for cardiovascular disorders } \\
\hline Cardiotonic drugs & $5(7.2)$ & $0(0.0)$ & $0.068^{\mathrm{a}}$ \\
\hline Amiodarone & $6(8.7)$ & $0(0.0)$ & $0.028^{a_{*}}$ \\
\hline Antianginal drugs & $5(7.2)$ & $9(13.0)$ & 0.259 \\
\hline Creatine phosphate & $4(5.8)$ & $3(4.3)$ & $1^{\mathrm{a}}$ \\
\hline Antilipemic agents & $26(37.7)$ & $10(14.5)$ & $0.002^{*}$ \\
\hline Anticoagulants & $16(23.2)$ & $6(8.7)$ & $0.033^{*}$ \\
\hline Gastrointestinal stimulants & $9(13.0)$ & $11(15.9)$ & 0.629 \\
\hline Probiotics & $17(24.6)$ & $15(21.7)$ & 0.687 \\
\hline Hepatic protectants & $15(21.7)$ & $6(8.7)$ & $0.033^{*}$ \\
\hline \multicolumn{4}{|l|}{ Drugs for respiratory disorders } \\
\hline Anti-asthmatics & $8(11.6)$ & $20(29.0)$ & $0.011^{*}$ \\
\hline Expectorants & 25 (36.2) & $32(46.4)$ & 0.226 \\
\hline NSAIDs & $14(20.3)$ & $8(11.6)$ & 0.163 \\
\hline Sedatives \& analgesics & $9(13.0)$ & $2(2.9)$ & $0.028^{*}$ \\
\hline RBC transfusion & $7(10.1)$ & $1(1.4)$ & $0.069^{\mathrm{a}}$ \\
\hline Albumin transfusion & $22(31.9)$ & $10(14.5)$ & $0.016^{*}$ \\
\hline
\end{tabular}

a, compared by Fisher's exact test. * $\mathrm{P}<0.05$. CCBs, calcium channel blockers; CVD, cerebrovascular disease; ECMO, extracorporeal membrane oxygenation; NSAID, nonsteroidal anti-inflammatory drug; RAAS, renin-angiotensin-aldosterone system; RBC, red blood cell; rhG-CSF, recombinant human granulocyte colony-stimulating factor. 
A

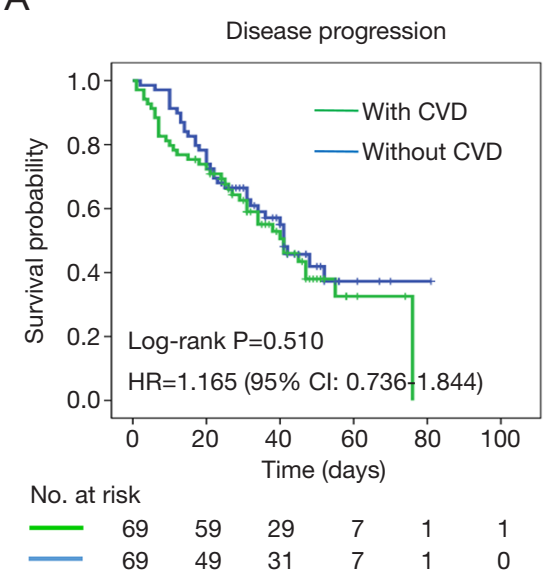

$\mathrm{D}$

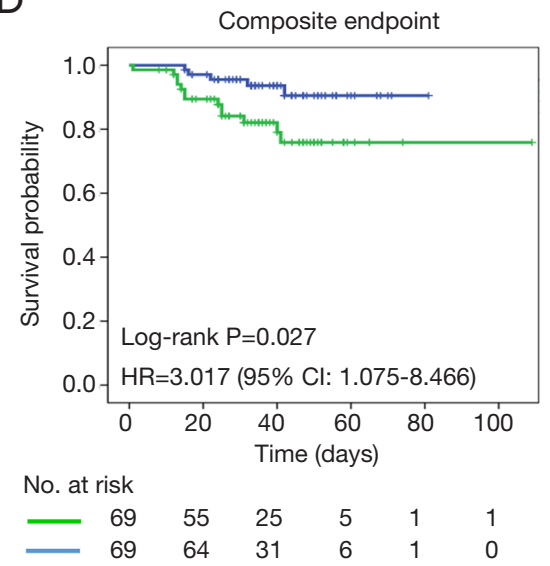

B

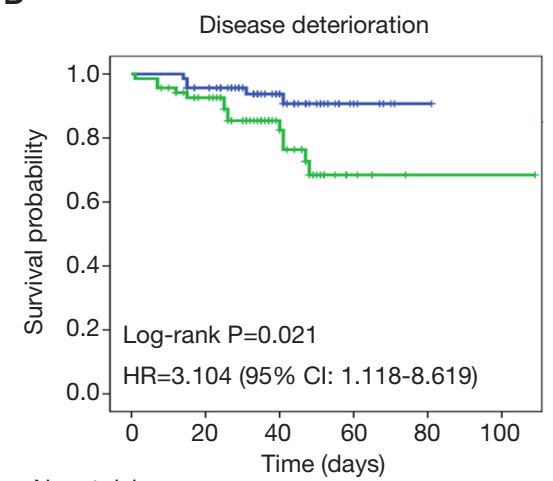

No. at risk

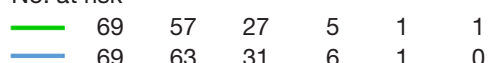

C

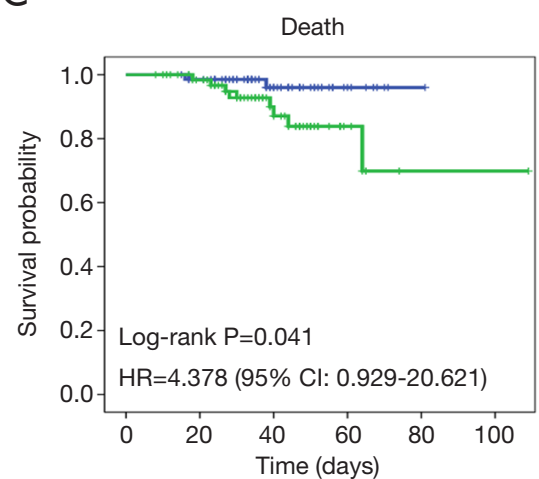

No. at risk

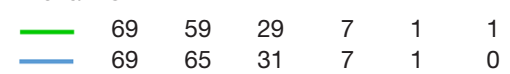

$E$

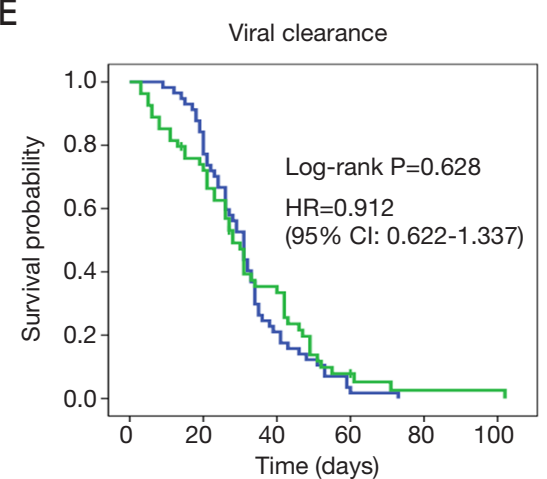

No. at risk

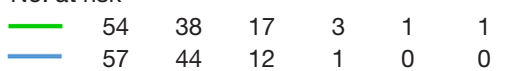

Figure 3 Kaplan-Meier plots for different clinical outcomes in patients with and without pre-existing cerebrovascular diseases. The figure displays Kaplan-Meier survival plots according to disease progression (A), disease deterioration (B), death (C), the composite endpoint (D), and viral clearance (E). CVD, cerebrovascular disease; HR, hazard ratio.

bacterials, mechanical ventilation, and ICU admission. Furthermore, therapeutics aiming at controlling and preventing comorbidities and complications were also more frequently administered to patients with pre-existing CVD.

Since the outbreak of the COVID-19 pandemic, neurological symptoms and complications of the disease have been widely described (34). COVID-19 has also been reported to be an independent predictor for the occurrence of stroke in hospitalized patients as well as mortality of these patients with stroke $(35,36)$. It is suggested that COVID-19 represents a state of hypoxia, inflammation, and hypercoagulability. These pathophysiological changes may underpin the development of stroke (37). However, comprehensive data concerning the impact of pre-existing CVD on the clinical course of COVID-19 have seldom been reported. To date, several cohort studies have compared clinical data between patients with and without pre-existing CVD. They observed that COVID-19 patients with preexisting CVD were older and more likely to be male, and had more comorbidities and severer disease than patients without CVD, resulting in a higher mortality rate (5-8). One limitation of these studies is that the authors lost sight of the impact of age and sex on the clinical course and outcomes of patients with COVID-19, since they had already been verified as independent predictors of adverse outcome of COVID-19 (16,17). Since the aim of our study was to explore the impact of CVD pathophysiology on the clinical course of COVID-19, a matching method was utilized to account for the impact of the demographic features on COVID-19. In addition to the risk conferred by age and sex, pre-existing 
Table 5 Cox regression analyses of risk factors for the outcome of the composite endpoint in matched COVID-19 patients with and without CVD

\begin{tabular}{|c|c|c|c|c|}
\hline Variable & \multicolumn{2}{|c|}{ Univariable analysis } & \multicolumn{2}{|c|}{ Multivariable analysis } \\
\hline Age, y & $1.016(0.966-1.068)$ & 0.539 & - & - \\
\hline Male & $1.496(0.561-3.987)$ & 0.421 & & \\
\hline Hypertension & $1.069(0.414-2.761)$ & 0.890 & & \\
\hline $\mathrm{CHD}$ & $0.279(0.037-2.099)$ & 0.215 & & \\
\hline CVD & $3.017(1.075-8.466)$ & 0.036 & $3.155(1.121-8.878)$ & 0.030 \\
\hline COPD & $1.040(0.239-4.524)$ & 0.958 & & \\
\hline Malignancy & $3.097(0.892-10.752)$ & 0.075 & - & - \\
\hline
\end{tabular}

The multivariable model contains age, CVD, and malignancy. CHD, coronary heart disease; CKD, chronic kidney disease; COPD, chronic obstructive pulmonary disease; CVD, cerebrovascular disease; HR, hazard ratio.

CVD itself was demonstrated to be associated with an unfavorable outcome of COVID-19 in the present study.

One interesting phenomenon we identified was that higher proportions of COVID-19 patients with pre-existing CVD were asymptomatic or presented with unapparent system symptoms (chill, myalgia, and fatigue) and respiratory symptoms (cough and dyspnea) at disease onset compared with their non-CVD counterparts. Accordingly, patients with pre-existing CVD were less likely to receive anti-asthmatic drugs during hospitalization. However, they were more likely to have severe disease and to deteriorate to a critical condition. Considering these observations, the possibility of severe illness masquerading as mild symptoms should not be overlooked, and much more attention should be paid to this subgroup of COVID-19 patients.

Additionally, COVID-19 patients with pre-existing CVD presented with clinical characteristics seen in severe or critical illness, including cytokine storm, ARDS, and subsequent extra-pulmonary organ dysfunction, as evidenced by abnormities in IL-6 and other laboratory indices during the disease's clinical course. Cell entry of SARS-CoV-2 directly damages the lungs, kidneys, heart, gastrointestinal tract, and vasculature (38). In most cases, it is self-limited, owing to the clearance of SARS-CoV-2 from the lung via adaptive immune response. However, in severe cases, an aberrant uncontrolled response, in the form of a "cytokine storm", can result in multiple organ failure (39). The key mechanism is that, in the late stage of COVID-19, cytokine storm induces endothelial damage, predisposes the thrombotic/fibrinolytic imbalance toward a status of microthrombosis and microcirculatory disturbance, and finally causes multi-organ ischemic or hemorrhagic complications, especially in the lungs, heart, and kidneys (38). Consequently, the use of therapeutics aimed at controlling secondary infection and multi-organ support have been emphasized for patients with severe COVID-19 $(21,24)$, as also seen in our study.

The pathophysiology of CVD is closely related to the specific clinical characteristics of the subgroup of COVID-19 patients included in this study. Firstly, due to bulbar paralysis, impaired locomotion, insufficient nutrition, and cognitive disorder, patients with pre-existing CVD have poor immunity and cardiac function, resulting in an escalated risk of secondary infection, especially hospital-acquired pneumonia $(6,40)$. Secondly, owing to the disturbance of central nervous system regulatory functions, patients affected by neurological disease have lower ability to compensate for COVID-19 with unapparent symptoms, which leads to severer infection and respiratory depression (5). Thirdly, as illustrated in this study, patients with pre-existing CVD are more likely to have other comorbidities, such as hypertension and diabetes, which themselves are associated with mortality from COVID-19 $(4,11,18)$. CVD may serve as a proxy for vascular frailty and a pro-inflammatory status caused by hypertension and diabetes, which predisposes patients to developing severer endothelial injury and subsequent multiorgan injury upon infection with SARS-CoV-2 (41). Last but not least, the potential neurotropism of SARS-CoV-2 could exert a possible detrimental effect in patients with preexisting neurological diseases $(42,43)$, which may form a vicious cycle for the pathophysiology of COVID-19. 
Concerning therapeutics, there are several important findings in this study. Firstly, more COVID-19 patients with pre-existing CVD received third-line antibacterials, corroborating the existence of severer secondary infection in this subgroup of patients. Secondly, owing to disease deterioration, more patients with pre-existing CVD required ICU admission, and subsequent use of mechanical ventilation, sedatives \& analgesics, and multiorgan supporting therapeutics. We observed more use of hepatic protectants, albumin, and amiodarone, as well as a trend toward more use of cardiotomic drugs in those with pre-existing CVD. However, no significance of other supporting treatments, such as creatine phosphate and renal replacement therapy, was seen between the two groups, which we believe may be owing to the small sample size of this study. Thirdly, greater proportions of patients with pre-existing CVD required antihypertensives, antidiabetics, antilepemic agents, anticoagulants, and antiplatelet agents. Hence, drugs targeting underlying diseases should also be emphasized in the management. These findings not only have important instruction significance for the treatment of this subgroup of patients, but also corroborate with the features of the clinical course mentioned above, which may deepen our understanding of the pathophysiology of comorbid COVID-19 and CVD.

There are several limitations to this work. Firstly, it was a single-center retrospective study with a relatively small number of patients, which made it challenging to accurately assess various risk factors using a multifactor regression model. Secondly, our findings may not be generalizable to other regions worldwide with diverse epidemiological characteristics, since all participants in this study were from the epicenter Wuhan during the early days of the outbreak. Thirdly, the available data did not address the heterogeneity of CVD, including disease severity and disease subtype, owing to the small sample size; future analysis with a larger population on a nationwide basis should consider these features as additional susceptibility factors. Fourthly, continued observation and follow-up of this subpopulation with COVID-19 is critical. Nevertheless, we hope that our results will provide guidance for clinicians to understand the complete picture of the disease and be conducive to improving the management of patients with COVID-19 who have a history of CVD.

\section{Acknowledgments}

We sincerely thank all patients who were involved in this study and their families, as well as all medical workers and civilians for their dedication and sacrifice in the fight against the virus.

Funding: This work was supported by the National Natural Science Foundation of China (grant No. 81971133 to GL and grant No. 81702999 to LL).

\section{Footnote}

Reporting Checklist: The authors have completed the STROBE reporting checklist Available at http://dx.doi. org/10.21037/atm-21-2237

Data Sharing Statement: Available at http://dx.doi. org/10.21037/atm-21-2237

Conflicts of Interest: All authors have completed the ICMJE uniform disclosure form (available at http://dx.doi. org/10.21037/atm-21-2237). LL serves as an unpaid editorial board member of Annals of Translational Medicine from Apr 2020 to Mar 2022. The authors have no other conflicts of interest to declare.

Ethical Statement: The authors are accountable for all aspects of the work in ensuring that questions related to the accuracy or integrity of any part of the work are appropriately investigated and resolved. This study was conducted in accordance with the Declaration of Helsinki (as revised in 2013) and approved by the National Health Commission of China and the Institutional Review Board of Huoshenshan Hospital (approval no. K202101-02). The requirement for written informed consent was waived by the ethics committees of the designated hospital for patients with emerging infectious diseases.

Open Access Statement: This is an Open Access article distributed in accordance with the Creative Commons Attribution-NonCommercial-NoDerivs 4.0 International License (CC BY-NC-ND 4.0), which permits the noncommercial replication and distribution of the article with the strict proviso that no changes or edits are made and the original work is properly cited (including links to both the formal publication through the relevant DOI and the license). See: https://creativecommons.org/licenses/by-nc-nd/4.0/.

\section{References}

1. Lu R, Zhao X, Li J, et al. Genomic characterisation and 
epidemiology of 2019 novel coronavirus: implications for virus origins and receptor binding. Lancet 2020;395:565-74.

2. Coronavirus disease (COVID-19) Weekly Epidemiological Update and Weekly Operational Update. Available online: https://www.who.int/emergencies/diseases/novelcoronavirus-2019/situation-reports. Date last updated: March 22 2021; Accessed: March 22, 2021.

3. Blagosklonny MV. From causes of aging to death from COVID-19. Aging (Albany NY) 2020;12:10004-21.

4. Guan WJ, Liang WH, Zhao Y, et al. Comorbidity and its impact on 1590 patients with COVID-19 in China: a nationwide analysis. Eur Respir J 2020;55:2000547.

5. Romagnolo A, Balestrino R, Imbalzano G, et al. Neurological comorbidity and severity of COVID-19. J Neurol 2021;268:762-9.

6. Yao X, Liu S, Wang J, et al. The clinical characteristics and prognosis of COVID-19 patients with cerebral stroke: A retrospective study of 113 cases from one single-centre. Eur J Neurosci 2021;53:1350-61.

7. Qin C, Zhou L, Hu Z, et al. Clinical Characteristics and Outcomes of COVID-19 Patients With a History of Stroke in Wuhan, China. Stroke 2020;51:2219-23.

8. Kummer BR, Klang E, Stein LK, et al. History of Stroke Is Independently Associated With In-Hospital Death in Patients With COVID-19. Stroke 2020;51:3112-4.

9. Du RH, Liang LR, Yang CQ, et al. Predictors of mortality for patients with COVID-19 pneumonia caused by SARS-CoV-2: a prospective cohort study. Eur Respir J 2020;55:2000524.

10. Bai $Y$, Wang E, Zhao S, et al. Implications of Laboratory Tests in Disease Grading and Death Risk Stratification of COVID-19: A Retrospective Study in Wuhan, China. Front Med (Lausanne) 2021;8:629296.

11. Wang B, Li R, Lu Z, et al. Does comorbidity increase the risk of patients with COVID-19: evidence from metaanalysis. Aging (Albany NY) 2020;12:6049-57.

12. Aggarwal G, Lippi G, Michael Henry B. Cerebrovascular disease is associated with an increased disease severity in patients with Coronavirus Disease 2019 (COVID-19): A pooled analysis of published literature. Int J Stroke 2020;15:385-9.

13. Patel U, Malik P. Pre-existing cerebrovascular disease and poor outcomes of COVID-19 hospitalized patients: a meta-analysis. J Neurol 2021;268:240-7.

14. Siepmann T, Sedghi A, Barlinn J, et al. Association of history of cerebrovascular disease with severity of COVID-19. J Neurol 2021;268:773-84.
15. Pranata R, Huang I, Lim MA, et al. Impact of cerebrovascular and cardiovascular diseases on mortality and severity of COVID-19-systematic review, metaanalysis, and meta-regression. J Stroke Cerebrovasc Dis 2020;29:104949.

16. Zhou F, Yu T, Du R, et al. Clinical course and risk factors for mortality of adult inpatients with COVID-19 in Wuhan, China: a retrospective cohort study. Lancet 2020;395:1054-62.

17. Chen T, Wu D, Chen H, et al. Clinical characteristics of 113 deceased patients with coronavirus disease 2019: retrospective study. BMJ 2020;368:m1091.

18. Shi Q, Zhang X, Jiang F, et al. Clinical Characteristics and Risk Factors for Mortality of COVID-19 Patients With Diabetes in Wuhan, China: A Two-Center, Retrospective Study. Diabetes Care 2020;43:1382-91.

19. Cortes Garcia M, Sierra Moros MJ, Santa-Olalla Peralta $\mathrm{P}$, et al. Clinical characteristics and outcomes of diabetic patients who were hospitalised with 2009 pandemic influenza A H1N1 infection. J Infect 2012;64:218-24.

20. Yang $\mathrm{X}, \mathrm{Yu} \mathrm{Y}, \mathrm{Xu} \mathrm{J}$, et al. Clinical course and outcomes of critically ill patients with SARS-CoV-2 pneumonia in Wuhan, China: a single-centered, retrospective, observational study. Lancet Respir Med 2020;8:475-81.

21. World Health Organization interim guidance. Clinical management of severe acute respiratory infection when novel coronavirus $(\mathrm{nCoV})$ infection is suspected: interim guidance. Available online: https://www.who.int/ publications-detail/clinical-management-of-severe-acuterespiratory-infection-when-novel-coronavirus-(ncov)infection-is-suspected. Published: January 28, 2020; Accessed: January 31, 2020. 2020.

22. Chinese Society of Neurology CSS. Chinese guidelines for diagnosis and treatment of acute ischemic stroke 2018. Chinese Journal of Neurology 2018;51:666-82.

23. Chinese Society of Neurology CSS. Chinese guidelines for diagnosis and treatment of acute intracerebral hemorrhage 2019. Chinese Journal of Neurology 2019;52:994-1005.

24. The National Health Commission of People's Republic of China. Interpretation of New Coronavirus Pneumonia Diagnosis and Treatment Plan (Trial Version 6) (in Chinese). Available online: http://www.nhc.gov.cn/yzygj/ s7652m/202002/54e1ad5c2aac45c19eb541799bf637e9. shtml. Accessed April 3, 2020. 2020.

25. Thygesen K, Alpert JS, Jaffe AS, et al. Fourth Universal Definition of Myocardial Infarction (2018). Circulation 2018;138:e618-51.

26. EASL Clinical Practice Guidelines: Drug-induced liver 
injury. J Hepatol 2019;70:1222-61.

27. Kidney Disease: Improving Global Outcomes (KDIGO) Acute Kidney Injury Work Group. KDIGO clinical practice guideline for Acute Kidney Injury. Kidney Int Suppl 2012;2012;2:1-138.

28. Iba T, Nisio MD, Levy JH, et al. New criteria for sepsisinduced coagulopathy (SIC) following the revised sepsis definition: a retrospective analysis of a nationwide survey. BMJ Open 2017;7:e017046.

29. Taylor FB, Jr., Toh CH, Hoots WK, et al. Towards definition, clinical and laboratory criteria, and a scoring system for disseminated intravascular coagulation. Thromb Haemost 2001;86:1327-30.

30. Wang L, He W, Yu X, et al. Coronavirus disease 2019 in elderly patients: Characteristics and prognostic factors based on 4-week follow-up. J Infect 2020;80:639-45.

31. Ranieri VM, Rubenfeld GD, Thompson BT, et al. Acute respiratory distress syndrome: the Berlin Definition. Jama 2012;307:2526-33.

32. Marshall JC, Cook DJ, Christou NV, et al. Multiple organ dysfunction score: a reliable descriptor of a complex clinical outcome. Crit Care Med 1995;23:1638-52.

33. Rhodes A, Evans LE, Alhazzani W, et al. Surviving Sepsis Campaign: International Guidelines for Management of Sepsis and Septic Shock: 2016. Crit Care Med 2017;45:486-552.

34. Ellul MA, Benjamin L, Singh B, et al. Neurological associations of COVID-19. Lancet Neurol 2020;19:767-83.

35. Katz JM, Libman RB, Wang JJ, et al. Cerebrovascular

Cite this article as: Bai Y, Liang Y, Wang F, Chen L, Zhao Y, Zhao S, Zhu Y, Dong Y, Liang G, Chen D, Liu L. Clinical course and outcomes of COVID-19 patients with a history of cerebrovascular disease: a retrospective study in Wuhan. Ann Transl Med 2021;9(12):988. doi: 10.21037/atm21-2237
Complications of COVID-19. Stroke 2020;51:e227-31.

36. Benussi A, Pilotto A. Clinical characteristics and outcomes of inpatients with neurologic disease and COVID-19 in Brescia, Lombardy, Italy. Neurology 2020;95:e910-20.

37. Fan H, Tang X. Influence of COVID-19 on Cerebrovascular Disease and its Possible Mechanism. Neuropsychiatr Dis Treat 2020;16:1359-67.

38. Libby P, Lüscher T. COVID-19 is, in the end, an endothelial disease. Eur Heart J 2020;41:3038-44.

39. Schett G, Manger B, Simon D. COVID-19 revisiting inflammatory pathways of arthritis. Nat Rev Rheumatol 2020;16:465-70.

40. Quyet D, Hien NM, Khan MX, et al. Risk Factors for Stroke Associated Pneumonia. Open Access Maced J Med Sci 2019;7:4416-9.

41. Sardu C, Gambardella J, Morelli MB, et al. Hypertension, Thrombosis, Kidney Failure, and Diabetes: Is COVID-19 an Endothelial Disease? A Comprehensive Evaluation of Clinical and Basic Evidence. J Clin Med 2020;9:1417.

42. Baig AM, Khaleeq A, Ali U, et al. Evidence of the COVID-19 Virus Targeting the CNS: Tissue Distribution, Host-Virus Interaction, and Proposed Neurotropic Mechanisms. ACS Chem Neurosci 2020;11:995-8.

43. Li YC, Bai WZ, Hashikawa T. The neuroinvasive potential of SARS-CoV2 may play a role in the respiratory failure of COVID-19 patients. J Med Virol 2020;92:552-5.

(English Language Editor: J. Reynolds) 